\title{
Peculiarities of the Impact of Heat Treatment on the Magnetic Sensitivity of Irradiated by Electrons Single Crystals $n$-Ge
}

\author{
S.V. Luniov, A.I. Zimych, M.V. Khvyshchun \\ Lutsk National Technical University, LNTU \\ 75 Lvivska Str., Lutsk 43018, Ukraine
}

\begin{abstract}
In this article has been investigated the isothermal annealing of irradiated by the flow of electrons $\Omega=5 \cdot 1015 \mathrm{el} . / \mathrm{cm} 2$, with the energy of $10 \mathrm{MeV}$, of single crystals n-Ge. Coefficients of the magnetic sensitivity for irradiated single crystals $n$-Ge before and after annealing at different temperatures were also determined. The abnormal annealing at the temperature of $403 \mathrm{~K}$ has been revealed. Hall constant was being increased during this process and the maximal magnetic sensitivity was being achieved correspondingly. Such abnormal annealing can be used as a tool to create highly sensitive Hall sensors on the basis of the irradiated n-Ge.
\end{abstract}

Index Terms-Isothermal annealing, crystals n-Ge, magnetic sensitivity, irradiated n-Ge, Hall constant.

\section{INTRODUCTION}

At present time the development of semiconductor sensors is one of the most perspective directions in progress of the measuring systems element base. Systems of management and control, measuring complexes and devices can not work without all sorts of converters of physical quantities into electrical signals which are called transducers or sensors [1-4]. The significant attention among such devices is given to the sensors of the magnetic fields. Their nomenclature changes constantly, which is primarily associated with the development of electronic equipment. In addition, measurements of the magnetic fields parameters find their application in both fundamental and applied researches [5]. For example, measurements of the magnetic fields and also the parameters of the magneto-conductive environments in the frequency range from zero to super high are the important problems of radio electronics [6]. Issues, which are concerned of the control of the plasma flows in the tokamak systems, require a special attention.

This control is carried out by the magnetic fields of the appropriate configurations. The magnetic field in itself is regulated by a system of transducers that have high thermal and radiation resistance $[7,8]$. Hall sensors are one of the most common types of the magnetically sensitive items, which are widely used in medicine, engineering, flaw detection, geology (in studies of new deposits), in a creation of systems positioning and navigation [4, 6, 9-13]. A wide range of measuring instruments of various types and designs for investigation of the parameters of magnetic fields is represented at the market of magnetic sensor technology nowadays. However, such advanced manufacturers of magnetic sensor devices as "Honeywell", "Asachi Kasei MicroSystems», «Infinlon Technologies», «Micronas», «Melexis N.V." prefer the semiconductor sensors [14, 15]. Such sensors, with sufficient accuracy for industrial applications, have small dimensions, relatively low price, easy in operation.

Intensive development of semiconductor physics and technologies of their obtaining contributes the improvement of properties the existing semiconductor materials, as well as the search and development of new semiconductor materials. The highly sensitive magnetic field sensors on basis of these materials can be created. The elementary semiconductors $\mathrm{Si}$ and Ge are relevant to these semiconductors materials, which are used as a raw material for the creation of Hall sensors [6].

As we know [16], physical properties of semiconductors can be significantly modified by the influence of irradiation of high energy particles or quanta of high energies and heat treatment. This allows to change and adjust them the required properties properties purposefully. And this, in its turn, can be the basis of technologies for creation of the semiconductor devices and sensors with set in advance properties. Also, research of the radiation defects annealing processes gives the possibility to set the conditions of stable operation of the equipment, which includes elements on the basis of the irradiated material. So, research of the radiation defects annealing processes in single crystals $n-G e$ and their impact on physical properties of single crystals $n-G e$ is interesting both from the theoretical and applied points of view.

\section{EXPERIMENTAL RESULTS AND THEORETICAL CALCULATIONS}

Influence of irradiation by the flow of electrons $\Omega=5 \cdot 10^{15}$ $e l . / \mathrm{cm}^{2} \mathrm{~cm}^{-2}$ with the energy of $10 \mathrm{MeV}$ and isothermal annealing on the magnetic sensitivity of single crystals n-Ge were investigated in the given work. Results of our previous researches $[17,18]$ show that the point defects (A-centers [19]) and regions of disordering were introduced for the same single crystals $\mathrm{n}-\mathrm{Ge}$ at the above-mentioned conditions of 
electronic irradiation. The author of the work [20] models point defect in Ge with the same energy spectrum as a complex, which consists of a vacancy, an atom of oxygen and two interstitial atoms of Germanium $\left(\mathrm{VOI}_{2 \mathrm{Ge}}\right)$. The isothermal annealing of the irradiated samples $n-G e$ at the temperatures of $403 \mathrm{~K}, 433 \mathrm{~K}, 448 \mathrm{~K}$ and the measurements of temperature dependencies of the Hall constant were carried out to study the influence of heat treatment on the magnetic sensitivity of the irradiated single crystals n-Ge. We've also obtained dependencies of Hall voltage $U_{H}$ from the induction of magnetic field $B$ in the range from 0 to $0,5 \mathrm{~T}$ for unirradiated and irradiated samples before and after annealing (fig. 1-4).

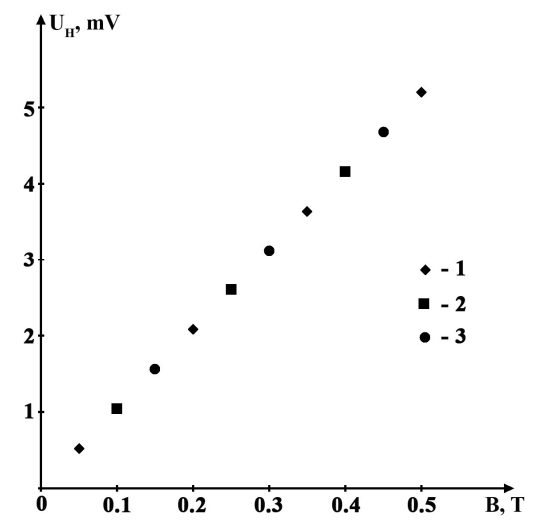

Fig 1. Dependencies of Hall voltage $U_{H}=f(B)$ for the unirradiated single crystals $\mathrm{n}-\mathrm{Ge}$ at different temperatures $\mathrm{T}, \mathrm{K}: 1-200 ; 2-250 ; 3$ -300 .

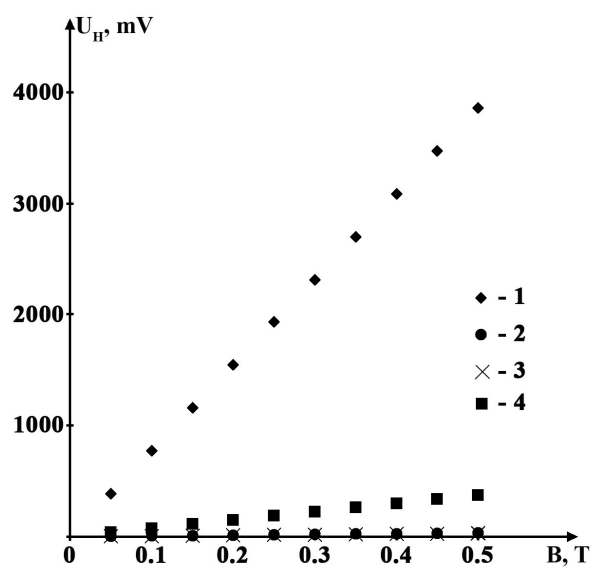

Fig. 2. Dependencies of Hall voltage $U_{H}=f(B)$ at $\mathrm{T}=200 \mathrm{~K}$ for the irradiated single crystals $\mathrm{n}-\mathrm{Ge}$ after the heat treatment within $1 \mathrm{~h}$. for different temperatures of annealing Tan, K: 1 - 403; 2 - 433; 3 - 448; 4 - unannealed sample.

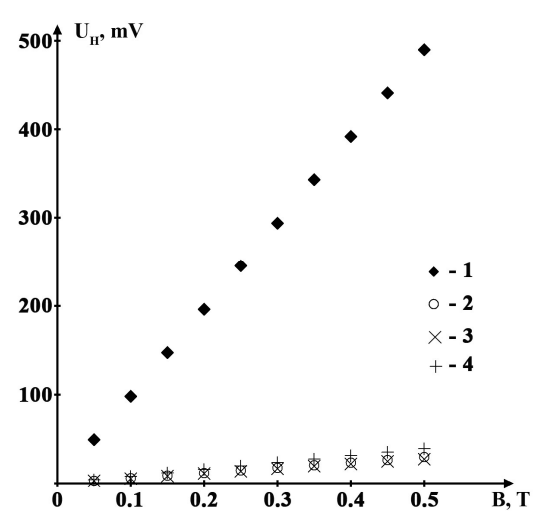

Fig. 3. Dependencies of Hall voltage $U_{H}=f(B)$ at $\mathrm{T}=250 \mathrm{~K}$ for the irradiated single crystals $\mathrm{n}$-Ge after the heat treatment within $1 \mathrm{~h}$. for different temperatures of annealing Tan, K: 1 - 403; 2 - 433; 3 - 448; 4 - unannealed sample.

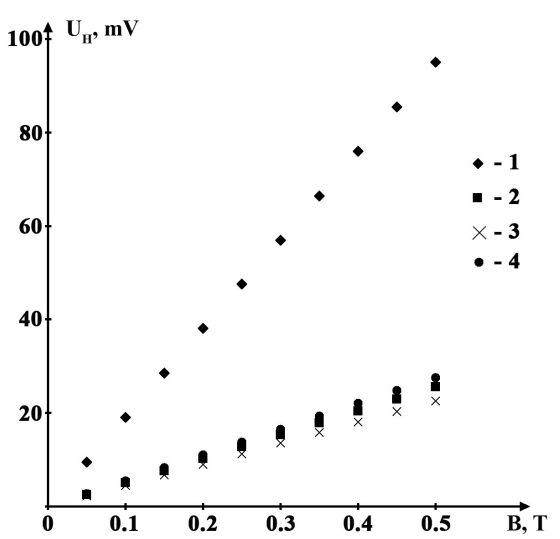

Fig. 4. Dependencies of Hall voltage $U_{H}=f(B)$ at $\mathrm{T}=300 \mathrm{~K}$ for the irradiated single crystals $\mathrm{n}-\mathrm{Ge}$ after the heat treatment within $1 \mathrm{~h}$. for different temperatures of annealing Tan, K: 1 - 403; 2 - 433; 3 - 448; 4 - unannealed sample.

When the direction of current and magnetic field are mutually perpendicular, then in accordance with [21] Hall voltage $U_{H}$ can be written as following:

$$
U_{H}=\frac{R_{H} I B}{d},
$$

where $R_{H}$ - Hall constant, $I$ - the force of current, which flows through the sample, $B$ - induction of magnetic field, $d$ - the thickness of sample in the direction of magnetic field. The magnetic sensitivity $\beta$ is one of the main characteristics of Hall sensors $[6,14,15]$ :

$$
\beta=\frac{\partial U_{H}}{\partial B} .
$$

If $R_{H}$ does not depend on the magnetic field, then the expression (2) can be written as:

$$
\beta=\frac{U_{H}}{B}=\frac{R_{H} I}{d} .
$$

Since the current $I$ and thickness of the sample $d$ can be defined, (for our case $I=1 \mu A, d=0,9 \mathrm{MM}$ ), the magnetic sensitivity will be also determined by the value of Hall constant $\mathrm{R}_{\mathrm{H}}$, which in its turn depends on the concentration of charge carriers and mechanisms of their scattering [21]. 
For the unirradiated single crystals $n-G e$, alloyed by the impurity $\mathrm{Sb}$ with a concentration $N_{d}=5 \cdot 10^{14} \mathrm{CM}^{-3}$, magnetic sensitivity of $\beta=10 \mathrm{mV} / \mathrm{T}$ does not depend on the temperature (fig.1) because the shallow donors of $\mathrm{Sb}$ are fully ionized. Under such conditions $\mathrm{n}-\mathrm{Ge}$ yields to other materials (which are used in the sensitive elements of Hall sensors) by value of the magnetic sensitivity to a considerable extent [6]. Because Hall constant $R_{H}$ is inversely proportional to the concentration of electrons in the conduction band [21] then, in accordance with (3), at the decrease of the extent of doping of semiconductors their sensitivity to the magnetic field will be increasing correspondingly. However, the control of impurities is one of the problems of the production, for example, of the especially pure germanium single crystals. Their content can be controlled only at the level of sensitivity of modern analytical instruments.

Dependencies of Hall voltage from the induction of magnetic field before and after isothermal annealing within 1 hour for single crystals $\mathrm{n}-\mathrm{Ge}$ at different temperatures are presented in fig. 2-4. All dependencies $\mathrm{U}_{\mathrm{H}}=\mathrm{f}(\mathrm{B})$ are linear in accordance with these figures. The facts indicate about minor role of the magnetoresistance. According to the works this effect can occur for single crystals of germanium with complexes that hold oxygen only at larger values of magnetic fields. Abnormal growth of magnetic sensitivity in the annealed samples in comparison with the unannealed was revealed after the isothermal annealing over a period of one hour at a temperature of $403 \mathrm{~K}$ (curves 1, fig. 2-4). The measurements of temperature dependencies of Hall constant for the annealed samples were conducted for a more detailed study of mechanism of the isothermal annealing and its influence on the magnetic sensitivity of the irradiated single crystals $\mathrm{n}-\mathrm{Ge}$. The value of Hall constant increased at a temperature of annealing $403 \mathrm{~K}$ in comparison with the irradiated (unannealed) samples. Increase of the annealing time to 3 hours at the temperature of $403 \mathrm{~K}$ leads to the growth of Hall constant and magnetic sensitivity (fig. 5).

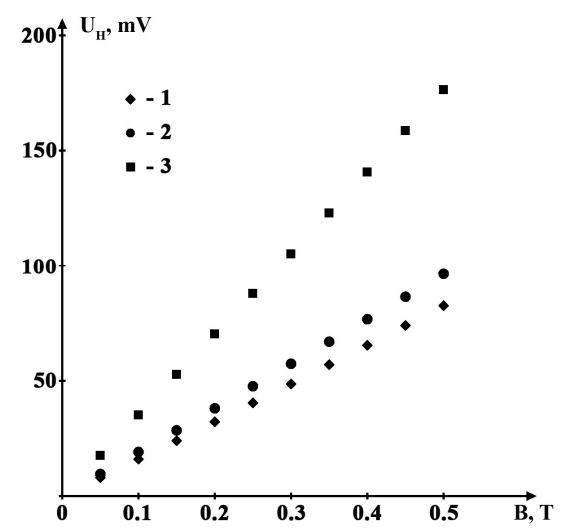

Fig. 5. Dependencies of Hall voltage at $\mathrm{T}=300 \mathrm{~K}$ for the irradiated single crystals $n-G e$ after the isothermal annealing at Tan $=403 \mathrm{~K}$, for different times of annealing tv, h.: $1-5 ; 2-1 ; 3-3$.

Further annealing to 5 hours leads to the decrease of Hall constant and magnetic sensitivity.
We also determined the parameters of radiation defects for the annealed samples because the change of Hall constant $R_{H}$ after annealing of the irradiated single crystals $\mathrm{n}-\mathrm{Ge}$ can be caused by both a change in concentration of the existing defects (A-centers, regions of disordering) and the emergence of new complexes with another energy spectrum.

Let the equilibrium concentration of radiation defects after the annealing in $\mathrm{n}$-Ge constituted $\mathrm{N}$ and for each such defect corresponds of $\mathrm{L}$ acceptor levels. Then the following equation of electroneutrality can be written for the temperatures when the shallow donors are fully ionized and the upper energy level of radiation defects is ionized partially:

$$
N(L-1)+n_{a}+n=N_{d},
$$

where $n_{a}$ - concentration of electrons at the highest on a scale of energies acceptor level, $n$ - concentration of electrons in the conduction band, $N_{d}$ - concentration of the donor's impurity. Taking into account the expressions for corresponding concentrations

$$
n_{a}=\frac{N}{2 e^{\frac{E_{a}-F}{k T}}+1}, n=N_{c} e^{\frac{F}{k T}},
$$

the equation (4) can be written as following:

$$
N(L-1)+\frac{N}{1+\frac{2 N_{c}}{n} e^{\frac{E_{a}}{k T}}}+n=N_{d},
$$

$N_{c}=\frac{\left(2 m_{n} k T\right)^{3 / 2}}{4 \pi^{3} \hbar^{3}}-$ effective density of states of the conductivity zone, $F$ - Fermi energy. Three unknown parameters of radiation defects appear in the equation (5). These parameters could have been changed in comparison with the irradiated (unannealed) samples: $N$ - concentration of radiation defects, $L$ - number of the acceptor levels that belong to each such defect, $E_{a}$ - ionization energy of the highest on a scale of energies acceptor level. In order to calculate of these parameters we write the equation (6) for three different values of concentration of electrons. As a result, a system of three equations was obtained. The above-mentioned parameters of radiation defects for the annealed samples $n-G e$ in different modes have been calculated, considering the obtained experimental results, value of the effective mass for density of states for electrons of the germanium conduction band and concentration of the impurity $\mathrm{Sb} N_{d}=5 \cdot 10^{14} \mathrm{~cm}^{-3}$. Parameters $L$ and Ea were the same as for irradiated (unannealed) single crystals n-Ge [17]. This fact indicates on impossibility or a small efficiency the formation of other types of radiation defects. The calculations show that concentration of A-centers decreases at the temperatures of annealing $433 \mathrm{~K}$ and $448 \mathrm{~K}$ and increases at the temperature of annealing $403 \mathrm{~K}$ to 3 hours.

\section{CONCLUSIONS AND DISCUSSIONS OF THE OBTAINED RESULTS}

The analysis of the obtained results shows that a heat treatment of single crystals n-Ge, irradiated by the high energy electrons, has a major impact on their sensitivity to the magnetic field. The magnetic sensitivity of the $n-G e$ at the 
temperatures of annealing Tan $=433 \mathrm{~K}$ and Tan=448 K decreases for the entire investigated range of temperatures, in comparison with the irradiated (unannealed) samples. This is explained by a decrease in the concentration of A-centers at annealing. Growth of the magnetic sensitivity for the entire investigated range of temperatures is observed at a temperature of annealing Tan $=403 \mathrm{~K}$ for annealing time up to 3 hours. As one can see from the theoretical calculations, under such conditions of annealing the concentration of Acenters in the annealed samples increases in comparison with the irradiated (unannealed) single crystals $n-G e$. As have explained earlier [18], irradiation of the same samples n-Ge by a flow of electrons $\mathrm{F}=5 \cdot 10^{15} \mathrm{~cm}^{-2}$, with the energy of $10 \mathrm{MeV}$, leads to the formation of A-centers and regions disordering. According to the Hosik model, the region disordering is a macroscopic inclusion of the spherical form with a large concentration of vacancies $\left(10^{18}-10^{20} \mathrm{~cm}^{-3}\right)$ in its kernel. Gradient of the vacancies concentration between the kernel of region disordering and its shell causes the diffusion of vacancies into the conductive matrix of single crystal n-Ge. The vacancies, which had been created at the annealing of kernels of regions disodering, may again react with oxygen and interstitial atoms of germanium and form new A-centers correspondingly.

Therefore, we can conclude that at heat treatment of the irradiated single crystals $n-G e$ the process of annealing occurs in parallel with the process of A-centers generation. Processes of the A-centers annealing prevail over the processes of their generation at the temperatures of an isothermal annealing Tan $=433 \mathrm{~K}$ and $\mathrm{Tan}=448 \mathrm{~K}$ within one hour. Generation of the A-centers occurs at a temperature of annealing Tan $=403 \mathrm{~K}$, for times of annealing to 3 hours. An increase of the heat treatment time $(\tan >3 \mathrm{~h})$ at a temperature of $\operatorname{Tan}=403 \mathrm{~K}$ leads to the decrease of the magnetic sensitivity. This effect can be explained by the decrease of the regions disordering concentrations and vacancies correspondingly, from which can be formed A-centers.

Thus, the highest possible concentration of A-centers at a temperature of annealing Tan $=403 \mathrm{~K}$ over a period of 3 hours is generated. This explains a significant increase of the magnetic sensitivity $n-G e$, the obtained at the room temperature, relatively to the unirradiated samples (from $\beta=10$ $\mathrm{mV} / \mathrm{Tl}$ to $\beta=350 \mathrm{mV} / \mathrm{Tl}$ ) at a strength of current $\mathrm{I}=1 \mathrm{~mA}$ through the sample and the Hall plate thickness in the direction of the magnetic field $\mathrm{d}=0,9 \mathrm{~mm}$. As far as the thickness of Hall detector in Hall's sensors is $0,2-0,3 \mathrm{~mm}$ as a rule, and current can vary from units to tens of milliamperes [6], then a change of the given parameters will allow to increase the magnetic sensitivity of the investigated samples in ten times.

That's why, n-Ge, which had been irradiated by the high energy electrons and passed the heat treatment additionally, can be a perspective material for creation of the highly sensitive sensors of the magnetic field on its basis. A high threshold sensitivity (owing to the high magnetic sensitivity) and higher (in comparison with unirradiated samples) resistance to the various types of radiation will be the main advantages of such sensors.

\section{REFERENCES}

[1] Ch. Schott, P. A. Besse, R.S. Popovic, Planar Hall effect in the vertical Hall sensor, Sensor Actuat A-Phys. 85, pp.111-115 (2000). https://doi.org/10.1016/S0924-4247(00)00328-9

[2] A.Danilov, Modern industrial current sensors. Modern electronics. 10, pp., 2004).

[3] A.F. Aleinikov, V.A. Gridchin, M.P. Tsapenko, Sensors (perspective directions of development), p.176. NSTU Publishing House, Novosibirsk, (2001).

[4] A.F. Kaperko, Analysis of the state, development trends and new developments of sensors of information converters of measurement, control and management systems, Measuring Technology, 1, pp.3-7 (1998).

[5] Y.H. Kahng, Y.W. Kim, M.S. Kim, W. Song J.-H. Choi, Detection of the superconducting transition and magnetic flux trapping in a niobium micro-ring by using micro-Hall sensors, Journal of the Korean Physical Society 69, p.1456 (2016).

[6] V.A. Antropov, L. Kh. Antropova, Application of Galvanomagnetic Phenomena in Semiconductors for the Development of Microwave Devices, Penza, p.166, PGU (2011).

[7] Ďuran, O. Hronová, J. Stöckel, J. Sentkerestiová, J. Havlicek, Magnetic measurements using an array of integrated Hall sensors on the CASTOR tokamak, Rev. Sci. Instrum. p.79, 10F123 (2008).

[8] J. Yang, J.W. Lee, B.K. Jung, K.J. Chung, Y. S. Hwang, Development of an internal magnetic probe for current density profile measurement in Versatile Experiment Spherical Torus. Rev. Sci. Instrum. p.85, $11 \mathrm{D} 809$ (2014)

[9] J.A. Tapia, A.L. Herrera-May, P.J. Garcна-Ramнrez, J. MartinezCastillo, E. Figueras, A. Flores, E. Manjarrez, Sensing magnetic flux density of artificial neurons with a MEMS device. Biomed Microdevices 13(2) pp.303-310 (2011).

[10] J. Lenz, S. Edelstein, Magnetic Sensors and Their Applications. IEEE Sensors Journal 6(3), pp.631 - 649 (2006).

[11] S. Tumanski, Modern magnetic field sensors - a review, Przeglad Elektrotechniczny, 10, pp.1-12 (2013).

[12] M. I. Bichurin, V. Petrov, R. Petrov, Yu. V. Kiliba, F. Bukashev, A.Yu. Smirnov, D.N. Eliseev. Magnetoelectric Sensor of Magnetic Field. Ferroelectrics. 280, pp.199-202 (2002).

[13] V.S Osadchuk, O.V. Osadchuk, O.P. Bililivska, O.M. Zhaglovskaya, Review of measuring device of galvanomagnetic fields, Bulletin of NTU"KhPI", 42, p. 948 (2012).

[14] K. Staroverov, Integral Hall Sensors of Honeywell. Electronics News, 1, pp.9-13 (2010).

[15] S. Sysoeva Magnetic field sensors. The spectrum of high-volume products from leading suppliers, Components, and technology, 1, pp. 1932, 2012).

[16] N. T. Gorbachuk, P. I. Didenko, Influence of a neutron irradiation on the characteristics of semiconductor measuring converters of temperature, deformation, magnetic field, Surface, 4, pp.57-58 (2005).

[17] S. V. Luniov, A. I. Zimych, P. F. Nazarchuk, V. T. Maslyuk, I. G. Megela, Radiation defects parameters determination in $\mathrm{n}-\mathrm{Ge}$ single crystals irradiated by high-energy electrons, Nuclear Physics and Atomic Energy, 17, p.47 (2016).

[18] S. Luniov, A. Zimych, P. Nazarchuk, V. Maslyuk, I. Megela, The impact of radiation defects on the mechanisms of electron scattering in single crystals n-Ge, J. Phys. Stud. 19, p.4704 (2015).

[19] [J. Fage-Pedersen, A.N. Larsen, A. Mesli, Irradiation-induced defects In Ge studied by transient spectroscopies, Phys. Rev. B. 62, pp.1011610125 (2000).

[20] P. Dolgolenko Modification of radiation defects in $\mathrm{Si}$ and $\mathrm{Ge}$ by background impurity, Nuclear Physics and Atomic Energy, 14, p.377 (2013).

[21] E.V. Kuchis, Galvanomagnetic effects and methods of their investigation, Radio and Communication, Moscow (1990). 